\title{
Behaviors and Patterns of Searching Care Among Fulani's Children Living in Rural Area, in a Context of Therapeutic Pluralism in the Ferlo, Senegal
}

\author{
Ndèye Marème Sougou ${ }^{1,2,{ }^{*}, \text { Gilles Boetsch }^{3} \text {, Anta Tal-Dia }}{ }^{1,2}$ \\ ${ }^{1}$ Department of Preventive Medicine and Public Health, University Cheikh Anta Diop, Dakar, Senegal \\ ${ }^{2}$ Health Institute of Development, University Cheikh Anta Diop, Dakar, Senegal \\ ${ }^{3}$ French National Center of Sciences Research, International Joint Unit Environment Health Societies, University Cheikh Anta Diop, Dakar, \\ Senegal
}

\author{
Email address: \\ nmsougou@hotmail.com (N. M. Sougou),ndeyemareme.sougou@ucad.edu.sn (N. M. Sougou) \\ ${ }^{*}$ Corresponding author
}

\section{To cite this article:}

Ndèye Marème Sougou, Gilles Boetsch, Anta Tal-Dia. Behaviors and Patterns of Searching Care Among Fulani's Children Living in Rural Area, in a Context of Therapeutic Pluralism in the Ferlo, Senegal. Social Sciences. Vol. 7, No. 4, 2018, pp. 152-160. doi: 10.11648/j.ss.20180704.11

Received: June 12, 2018; Accepted: July 12, 2018; Published: July 23, 2018

\begin{abstract}
The diversity of therapeutic offers is a proven fact in the contemporary world. In a still traditional society that is the Ferlo, there is a therapeutic diversity that encompasses a wide range of people in a nomadic community. In a context of medical pluralism, this article was contributing to the comprehension of how the structuring of social structures and health systems and the inter-functionality of its components can influence therapeutic remedies. This was a case study. The case studied was the logic of care-seeking behaviour of deseased children in a context of therapeutic pluralism. A total of 30 peoples were interviewed. These included 20 mothers and caregivers aged 6 to 59 months, 8 traditional healers and 2 health providers from the Widou thiengholi health post. Semi-directive interview guides were used for the survey. Interviews with informants were conducted in the local language of the participants. The data obtained through each in-depth individual interview were analyzed separately through the thematic content analysis. Data analysis was performed using NVivo 11 software. In the Ferlo, in the gender relations in the management of the disease in the child, the grandmother had a predominant place in the therapeutic decision-making process. The representations of diseases allowed a binary typology of diseases into "doctors" and "healers" diseases. These representations determined the type of recourse. There were other reasons for the choice of the type of remedy. The quality of the caregiver-patient relationship was one of the main reasons that influenced the choice of the health care facility as a use of care. The poor quality of the reception and the bad behavior of the providers discourage the communities to go to the health post. Despite the fact that the communities recognize the effectiveness of the treatment provided there. This study showed that disease representations play an important role in defining care-seeking behavior. Thus the "singular representations" relating to the types of illness condition the type of recourse to care. Nevertheless, there were characteristics specific to a type of remedy which contributed to the underutilization of this type of remedy. In this study, it was the quality of the care-patient relationship that was judged to be of insufficient quality and that was a factor of the underutilization of modern health services.
\end{abstract}

Keywords: Searching Care, Representation of Illness, Caregiver-Patient Relationship

\section{Introduction}

Traditional medicine is the oldest form of medicine. The traditional care system before colonial times was the only care system in Africa. Even today, despite modern health care systems, traditional medicine is still the dominant health care system in Africa for millions of people in both rural and urban areas [1]. Modern health services are suffering from this situation and there is therefore under-utilization of these 
services by the populations. The concept of therapeutic itinerary describes in concrete terms the construction of therapeutic choices based on the different paths that patients and their families follow [2]. The therapeutic choice is not considered only as the recourse to a set of possible resources more or less formalized (but tending however to be it) in a given cultural space which would articulate the "biomedical" or the "cosmopolitan" and the "other medicines" (hear here all the specialists). It will integrate the domestic universe as the first place of the itinerary that shapes subsequent orientations [3]. The diversity of therapeutic offers is a proven fact in the contemporary world. It refers to a cosmopolitan therapeutic space. Therapeutic space is understand as being all groups, resources, practices, knowledge, representations, and symbols available to any society to face the problem of the maintenance of life and its maintenance for an implicit or explicit purpose of well-being or health [3]. The use of a socio-anthropological approach, taking into account cultural aspects to integrate them into the clinic, has been recommended for over twenty years by Kleinman (1980) [4].

The objective of this study is to understand the rationale behind therapeutic itinerary choices in rural areas in a nomadic Fulani community. In a context of medical pluralism, this article is contributing to the comprehension of how the structuring of social structures and health systems and the inter-functionality of its components can influence therapeutic remedies.

\section{Methods}

\subsection{Framework for the Study}

The study took place at Widou Tiengholi in May 2017, in the administrative department of Linguère. Widou Thiengoli is a Human-Environment observatory, seat of the implantation of the Sahel green wall. Widou Thiengoli is composed of a predominantly Fulani population.

\subsection{Type of Study}

This was a case study. The case studied was the logic of care-seeking behavior of sick children in a context of therapeutic pluralism.

\subsection{Populations}

The informants were mothers and caregivers of children aged 6-59 months, traditional healers and health providers from the Widou Thiengholi health post.

\subsection{Sampling}

This was a reasoned choice sample for mothers and caregivers and claimants. For traditional healers, a network sampling strategy was used. Mothers and caregivers were found at the household level. Data collection continued until data saturation.

Mothers and caregivers aged 6-59 months

In-depth individual interviews were conducted with mothers and caregivers of children aged 6-59 months.

These were 20 randomly selected legal guardians who were caring for children aged 6 to 59 months. The sample consisted of 5 fathers with an average age of $45.6 \pm 2.8$ years, 10 grandmothers with an average age of $52.3 \pm 4.9$ years, 4 mothers with an average age of $29.5 \pm 7.5$ years and one step-mother.

\section{For traditional healers}

A total of 8 in-depth individual interviews with traditional healers were conducted. There were 5 male and 3 female healers with an average age of 67.75 years. Four of these healers practiced their profession in a nomadic position.

Health care providers

Two (2) individual interviews had been conducted with health providers in Widou: a community relay (Woman) and the pharmacy agent at the health post (Male).

Table 1. Distribution of interviewees according to their characteristics.

\begin{tabular}{llll}
\hline Actors & Characteristics & N of interviews & Total Interviews \\
\hline \multirow{4}{*}{ Guardians of children 6 to 59 months } & Mother & 4 & \\
& Father & 5 & 20 \\
& Grand mother & 10 & \\
\multirow{3}{*}{ Traditional healers } & Step mother & 1 & 8 \\
\multirow{3}{*}{ Health workers } & Male & 5 & 2 \\
& Woman & 3 & \\
& Community Relay Providers (Women) & 1 & \\
& Pharmacist health post (Male) & 2 & \\
\hline
\end{tabular}

\subsection{Data Collection Strategy}

\section{Mothers and caregivers}

The selection of individuals to be interviewed took place on the day of data collection, using the nonprobability "random walk" sampling method. A geographic location was selected and interviewers followed a specific path to identify applicable homes. Every third house on this road had been screened to determine if a child aged 6 to 59 months lived there.

\section{Traditional healers}

Recruitment had been done through the network strategy. A guide living in Widou had helped to find a traditional healer who helped to find the other traditional healers. Gradually the interviewees had made it possible to establish contacts with other informants. 


\section{Health care providers}

Claimants who were present on the day of the survey were surveyed.

Survey tools

Semi-directive interview guides were used for the survey

\subsection{Data Collection}

Key informant interviews were conducted in the local language of the participants. The interviews were recorded electronically. Notes had been taken by show of hands to facilitate triangulation of the transcribed data.

\subsection{Analysis}

Each in-depth interview was analyzed to determine: representation of the disease among children in nomadic Fulani environments the reasons for therapeutic choices therapeutic itineraries the place of the health structure within the health care system.

The data obtained through each in-depth individual interview were analyzed separately through the thematic content analysis.

Data analysis was performed using NVivo 11 software.

\subsection{Validity}

For the validity of accessibility construct, questionnaires and interview guides were administered in Fulani language.

Triangulating data sources (child guardians, traditional healers and health providers) were made for the scientific validity.

\section{Results}

The range of types of care available in the Widou locality includes traditional medicine, self-medication and modern medicine through the existence of a care system attached to the Widou health post.

\subsection{Type of Disease by Population Perception}

The populations interviewed distinguish two types of diseases in children. These diseases were either called "doctor's diseases", i.e. those with symptoms that must be treated by the modern health care system, or "healer's diseases", i.e. those with symptoms that must be treated by the traditional health care system.

\subsubsection{Doctors' Diseases}

The "doctor's" diseases were grouped into a set of symptomatology that must be managed at the health post level. These are stomach aches, diarrhea and fever. Fever had a special place in the categorization of "physician's" illnesses. Her presence alone guided parents towards the use of health facilities.

PB 39 years, Mother of AK 14 months:....yes we consider as doctor's diseases, all the diseases that manifest themselves by fevers, stomach aches, diarrhoea."

For most interviewees, winter/rainy season would be responsible for many diseases because of runoff, for others the cause would be the presence of mosquitoes.

MSS, grandmother of SS 2 years old: Stream water is a factor of malaria. This water is harmful to children because it is not healthy."

SK, AK's grandmother, 14-month-old daughter: Mosquitoes bring this disease. If they sting you, you'll get sick."

Illnesses were also said to be linked to poor nutrition or "bad care" given to the child by his mother. Some parents believed that stunting may be related to poor nutrition.

$R S$, Father of $A S$, 5-year-old boy:... children's stunting may be related to poor or inadequate nutrition in the child. We live on food without vitamins. We live on couscous, rice with cowpeas. And, these foods don't have vitamins..."

\subsubsection{Healers' Disease}

Diseases known as "healer's diseases" had as the weight loss symptom (weight loss or overweight) in most cases. Seizures were also cited as symptoms of "healer" diseases. These diseases would have mystical causes and must be care for by healers. These diseases were complicated and even if they were treated in hospital, they would not be cured... They were caused by "social" phenomena that are beyond the understanding of modern medicine. These are mystical, hereditary or dietary causes such as suckling the milk of a pregnant woman.

GS, Mother of OS, 5-year-old daughter:"There is also the Bësgu" which can contribute to the child's lack of development. Sometimes traditional medicine is used... This disease prevents the child from growing."

MB Grandma, 45, grandson Oumar, 3 years old: "This weight does not belong to her body... The overweight I am talking about is related to epileptic seizures.... This is how in a Fulani environment, children who are overweight are explained."

Several reasons were cited as the cause of "healer" diseases. It's mostly about "rap" demons. Some of these diseases called "progeny" diseases would be hereditary.

$K A$ 58, NF grandmother, 5-year-old daughter.".there are demons haunting the child... If the person is confronted with these kinds of diseases, the hospital does not intervene. In this respect, people refuse to go there. Sometimes it's the child himself who refuses to go to the hospital thinking he's afraid."

RS, Father of AS, 5-year-old boy:... there are hereditary diseases. And most of it is transmitted by the mother."

\subsection{Typology of the Therapy Organizing Group}

In most of the families surveyed, there was a consultation process when the child is ill. It is often the mother who informs the family about the disease. The leaders of the therapy organizing group are often the grandmother and father. It is the father who often pays for the child's care, so he must be approved before taking the child to hospital. The important role of the influential grandmother in the child's care process was reported by most respondents. This role is 
all the more important as some interviewees point out that men are nomadic and often transhumant.

POS, grandmother, child 4 years old: "In any case, when his father is there, he is warned before the child goes to the dispensary."

First resort care

The two types of care that were used in first-line care were post health care and self-medication.

The preference of the health post was linked to the geographical accessibility of the health post.

MN Femme, 32, mother of Fatoumata Ba, a 3-year-old girl: "First of all we think of the doctor, before taking him elsewhere... When my child is sick, I take him first to the dispensary, before going elsewhere."

The types of self-medications used were mostly composed of drugs such as paracetamol and various types of syrups purchased in pharmacies or markets and kept in homes.

$M B$, Grandma, 45 years old, grandson Oumar Sy, 3 years old:... we have paracetamols for the first moments of these diseases... We bought them in the weekly markets."

Few parents go to first-line traditional healers unless the disease is deemed to be a "healer's" disease from the outset.

Second use of care

The choice of the second recourse is an alternative to the therapeutic failure of the first recourse to care. It was conditioned by the nature of the first recourse. As soon as the child does not get better despite the first-line treatment, they turn to another type of recourse.

Third recourse to care

The third recourse to care is often another health structure than the one they usually frequent. In this new structure, they will be able to benefit from better care. It can be the "big hospital" or another health post. The reported causes of the change in health structure are the referral by the "Widou doctor", the therapeutic failure of the treatment prescribed by the Widou doctor, the absence of the Widou doctor or the lack of medication at Widou's post.

$M N$, Woman, 32 years old, mother of FB, a 3 year old girl: Yes when you know that the doctor is not there or that certain medicines do not exist there, you go directly to Dara. Here, most choose Dara's great hospital."

\subsection{Treatment in Widou}

In the discourse of the inhabitants, 2 types of care are distinguished by their primacy in the choice by the inhabitants of Widou. These are self-medication and the use of modern health care services (health post).

\subsubsection{Place of Self-medication in the Care System in Widou}

There are two types of self-medication, one based on modern medicines (Efferalgan, paracetamol etc.) and the other based on traditional medicines (herbs and powder).

The type of drugs used in self-medication are in most cases drugs treating symptoms such as fever. These drugs are often purchased at the pharmacy, at the health station (public pharmacy) or at the weekly market. They are kept at home and given as first aid in case of illness. These are often paracetamol or other types of medications such as antibiotics and "rubbed" ointments.

SD, 23, mother of an 18-month-old boy: "They are given paracetamol but not traditional medicines. We buy them at the Widou, Boubacar or Amali Pharmacy."

Some interviewees report traditional self-medication. It is done using plants or powders used to relieve the first symptoms of the disease.

$R S$, 50, father of a 5-year-old child: We have plants and natural and medicinal powders to soothe diseases before going to the clinic."

\subsubsection{Place of the Health Structure Within the Health Care System}

Reasons for choosing a health facility as a health care provider

The reasons leading to the choice of the health structure as a place of care are the quality of the care-patient relationship, the positive perception of the health services offered by the health post, the existence of a deferred payment system.

Positive perception of health services

Most respondents have satisfaction points regarding the care received in health facilities. The most frequently cited reason for satisfaction is the existence of a deferred payment system. Thus most say that receiving loans when they need care is a positive point of the health structure. Other positive points noted by the interviewees were the perception of the positive impact of the care provided on their child's health on the one hand and the positive impact of vaccination on children's health on the other. For them, vaccination makes it possible to meet health personnel and has made it possible to eradicate certain diseases.

PB, 39, AK's mother, 14-month-old daughter: "But the doctor knows me and gives me loans evidenced by a paper and I repay when I can. That's why I prefer it there."

$F B$, 50, BS grandmother, 18-month-old boy: "We thank God, since the vaccinations came, the children no longer suffer from this disease".

Most healers in Widou have a good perception of modern health care. For the most part, they recommend that parents go to the doctor's office as a first resort in the event of a "doctor's" illness.

S, Femme, 54: "Modern medicine, I think it's a very effective medicine, managed by experienced people. Patients see themselves there and go there to get treatment."

The effectiveness of modern medicine is recognized by most healers. According to them, the diagnostic tools (TDR, X-ray, scanner, blood tests, etc.) available in modern medicine are effective. These diagnostic tools are the added value of modern medicine over traditional medicine.

Reference system

However, there are links of collaboration between Widou's traditional therapists and the health workers at the health post. This collaboration manifests itself in a reference of cases of diseases according to whether they are "doctors' diseases" or "healers' diseases". Cases are also referred from one system of care to another according to the therapeutic 
failures of one or the other of these systems. AGS, male, 76 years old: For me, as a traditional therapist, if I see that it is not a traditional disease, I take him to the hospital...."

Barriers to the choice of health care facility as a use of care

Barriers to the choice of health structure as a health care use are problems in the provider patient relationship, positive perception of health services offered by the health post, geographic accessibility, availability of medications and affordability.

The quality of relationships with health personnel can lead to attendance at the facility or, on the contrary, to visits to other health facilities, according to the interviewees. The inhabitants of Widou were often confronted with different types of problems concerning the caregivers of the Widou health post. These problems concerned the quality of the care-patient relationship and the availability of health personnel.

The caregiver-patient relationship appeared to be a conflict in most cases. At the welcoming stage to the health structure, some report behavioral problems of certain health personnel making access to health care services difficult. Tense relations between carers and carers were reported by the interviewees. These relationships influence attendance at the health post.

$R S$, grandmother, child 5 years old:"... there are many doctors in Widou but the problem is that they are not nice and it is very difficult but there are men and women and they are very difficult because we come, we are very sick and they are inside lying down and he does not even look at us. And if they get up again, they're angry at us and they give us a prescription that we can't even buy."

Interviewees complained in most cases about the availability of human resources. This could be due to an absence of staff or a lack of competence of health personnel. Caregivers were often absent from their workstations. For example, they could travel and not found the caregivers at the health station or found them tardively.

This availability was reflected in the therapeutic follow-up. Sometimes, according to the interviewees, there may be a follow-up problem leading to a late diagnosis or a tossing and turning between hospitals. Thus, some interviewees considered that the other hospitals were more competent than those in Widou. They speak of large "hospitals" where diagnosis in a higher technical plateau is better. In these large hospitals, caregivers communicate by explaining their illness.

MSD, the school principal, 45, His daughter FD, 4. ....here the treatment is superficial, the real treatment is in the big hospitals."

$A B G, 45$ years old father child age 2 and a half: Because, even if we bring a person who is in a critical situation, we can stay for a very long time without seeing the health personnel. You can also come very early in the morning to Widou health post and stay until 1lam without seeing the health staff. Really, it's our health post, but access is difficult."

Drug ruptures, especially in children's medications (syrup), were reported by interviewees.

AS, Ngolko 42 years old grandmother age child 2 years old: 'At the level of the health district of Widou, there is all the time of rupture of drugs."

Financial Accessibility

Problems concerning the high cost of care were highlighted by most interviewees. It manifests itself especially during the purchase of prescribed drugs. These are considered expensive for local residents. Often they sell their cattle to face the expenses of care.

$M D$, Father of AS, 4-year-old boy: "Most clinics prescribe us expensive drugs. The price of a goat or a sheep cannot buy them. You have to sell a cow to cover these drugs."

For most of the inhabitants, there are no financial coverage mechanisms for children. For example, most say they do not receive financial assistance for childcare.

GS, Mother of OS, 5-year-old daughter: "Here even the students pay. Even the newborn when you buy him a syrup, he pays... The ticket is 100fr. which is why we're asking for help because the prescription is more expensive than rice to eat."

$K A, 58$, grandmother, 5-year-old daughter: "Sometimes if the prescription costs 500 CFA francs, they have to increase it to 1000 francs to be able to pay the workers. Even tickets are like that."

Geographical accessibility problems have been reported. These problems lead to the use of means of transport such as carts.

FB, 50 years grandmother boy 18 months: "the dispensary is too far. It's 13 kilometers. Sometimes to bring a patient, you have to wake up early in the morning but with the transport problem, you are late. That's why we're boarding around noon....In these circumstances one tries to rent a car at 30000fr in the Wolof or Peul neighbourhood to go to Richard Toll or Dagana in order to undergo care under the endorsement of the existing nursing staff."

\subsubsection{Place of Traditional Medicine Within the Health Care System}

Reason for choosing traditional medicine

According to most traditional therapists, traditional medicine is a traditional heritage that must be respected. It is part of the Fulani culture and is a guarantee of roots to the culture. According to them, the villagers worthy of the name respect traditional medicine. It is effective and has many benefits, especially for children's health.

The reasons for choosing traditional medicine are linked to the type of diseases on the one hand and the therapeutic failure of modern care on the other.

POS, 48 years old grandmother child 4 years old: "We will go and see them when the doctors are incompetent in the face of the disease".

According to the interviewees, there are traditional child therapists. The latter treat diseases whose causes and treatments call upon ancestral knowledge inherited from the elders.

POS, 48 years old grandmother child 4 years old: "We choose a healer because he receives sick children. In addition, there are two healers who specialize in sick 
children; they are called thothers of children."

$P B, 39$ years old, Mother of AK, 14 months old daughter:

"These are very complicated pathologies to treat, they don't like medical treatment. Children suffer from these pathologies, we do not take them to doctors".

There are different types of traditional medicines in Widou. These range from incantations, talisman to powders and plants. In Widou, plants and powders are the most used in traditional treatments these plants are used some with Koranic verses or with amulets such as cords.

AS, Ngolko 42 years old grandmother age child 2 years old: These are medicines based on roots transformed into well sifted powder that we give to drink to the child before taking him to see a doctor. We use a small spoon that we give to drink to the child and we take a little to make the maceration... It is the elders who prepare them, our grandparents. You know in this village, there have been people since their ancestors, they are responsible for providing care. If we have a sick child, we go to see them."

We note that there are traditional means of prevention proposed by traditional medicine. These are mostly grey and talisman. Used for various prevention functions, they are widely used in the prevention of teething pain in young children.

The positive perception of traditional care is based on the ease of payment for care and the results of treatment in this case healing.

Some healers do not ask for anything for their payment. Others ask for small sums or modest gifts as payment.

POS, 48 years old grandmother child 4 years old: With healers, it's a negotiation. They don't impose anything; they offer prices and we discuss them. There are fewer difficulties with healers and they are not rare enough."

\section{Discussion}

Care-seeking behavior calls on individual determinants such as cultural values, psycho-social determinants and also the nature of the therapeutic offer available. The therapeutic space is the set of groups, resources, practices, knowledge, representations, and symbols that any society has at its disposal to face the problem of the sustenance of life and its maintenance with an implicit or explicit aim of well-being or health [5]. In Widou Thiengholi, there is a range of therapeutic remedies that include modern and traditional selfmedication, traditional medicine with its diversity of practice, modern medicine through the existence of the health post.

\subsection{Therapy Organizing Group}

Therapeutic redress decision-making mechanisms are collective and involve a "group of decision-makers" who will assess the need for the remedy and the nature of the remedy and/or fund the remedy. This "group of decision-makers" varies in size and composition depending on the social context, family structure, illness, the amount to be mobilized, the place of the patient in the family, and the financial autonomy of the patient or the child's mother [6]. In Widou, this organizing group is heterogeneous and is essentially composed of mother, father and grandparents.

\subsection{Gender Role in Therapeutic Decision-Making}

In most of the families surveyed, it is realized that there is a consultation process when the child is ill. It is often the mother who informs the family about the disease. The leaders of the therapy organizing group are in most cases the grandmother and father. Respondents report that it is often the father who pays for the child's care, so it requires his approval before taking the child to hospital. The important role of the grandmother as an influencer in the child's care process was reported by most respondents. This role is all the more important as some interviewees point out that men are nomadic and often transhumant.

PS AS grandmother, 14-month-old daughter. "When a child is sick, it is his mother who comes to inform us. You go see the child then, if we are critical, we take him to the hospital. If his father is there, he pays for it, if not, I pay for it."

In Senegal, the reduction in the role of mothers in favor of grandmothers had been emphasized in the use of therapeutic decisions in young children [7]. Indeed, in this study, it was proven that the grandmother was often the leader of the therapy organizing group when the child was sick and that the latter were often at the origin of the delay in using the health structure. In Nigeria, studies had shown that in West African patriarchal systems the decision-makers of the groups organizing therapies were often men and old women [8].

\subsection{The Therapeutic Itinerary Followed in Case of Illness in Children in Widou}

The concept of therapeutic itinerary describes in concrete terms the construction of therapeutic choices based on the different paths that patients and their families follow [2]. The therapeutic choice is not considered only as the recourse to a set of possible resources more or less formalized (but tending however to be it) in a given cultural space which would articulate the "biomedical" or the "cosmopolitan" and the "other medicines" (hear here all the specialists). Therapeutic choice is determined by cognitive models that have long been studied [9]. According to those authors, the first therapeutic remedy is explained by belief schemes subject to the need expressed and the benefits and risks associated with the treatment [9]. In this study, the first therapeutic choice is often self-medication. There are two types of selfmedications, one based on modern medicines (Efferalgan ${ }^{\circledR}$,

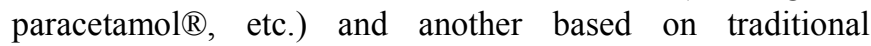
medicines (herbs and powder). Some interviewees report traditional self-medication. It is done using plants or powders used to relieve the first symptoms of the disease. $R S, 50$, father of a 5-year-old child: We have plants and natural and medicinal powders to soothe diseases before going to the clinic". Other studies had shown that self-medication was often the first and most obvious intention in cases of illness 
[10].

According to some respondents, the choice of health structure is determined by the relations maintained with health personnel. According to them, the quality of relations with health personnel can lead to attendance at the structure or, on the contrary, to travel to other health structures. Other interviewees also noted that the reason for choosing one health position over another is determined by its accessibility.

The reasons for choosing traditional medicine are linked to the type of diseases on the one hand and the therapeutic failure of modern care on the other. but there are reasons for the choice of modern medicine in relation to its financial accessibility.

Weiss MG al (1986) founded in their study, the existence of numerous recourse to traditional care before consulting modern care recourse. This study had shown also, a supremacy of traditional care recourse over modern care recourse in India [11]. This is not the case in this study, where there is a preference for modern care over traditional care. The results of this studies are similar to those of Joly P and al (2005) in Guadeloupe [12].

\subsection{Representation of Diseases: A Typology of Representations that Determines the Types of Care Sought for the Management of the Disease}

A medical, social and economic phenomenon, the notion of disease covers multiple dimensions. To account for this, Fabrega (1977) distinguishes the notions of "Illness", "Disease" and "Sickness", respectively understood as the subjective experience of something abnormal, the expression of a state of objective biophysical alteration and the sociocultural dimension of the disease [13]. This refers to the "Disease" of the disease in its biological sense, to the "illness", to the disease or the subjectively lived state of pathological disturbance, i.e. the perceptions and experiences lived by the individual with regard to health problems, and to the "Sickness", to the socialized, socially perceived and thought-out disease: social representation and symbolic burden by the social group as a whole [14].

The social function of the representations of the disease and in particular of its interpretation shows that the intellectual management of the event-disease has not only a social control function but, more widely, a social reproduction function [15].

The typology of diseases depends on the socio-cultural representation system of diseases.

In Widou, there are 2 types of diseases, "healers' diseases" and "doctors' diseases". The dichotomy found between the types of disease has been found in the literature where, "singular representations" are constructed from a fairly wide range of common representations on which everyone agrees [16].

This is the case in Widou, where "singular representations" involve the types of care sought for the management of the disease. Thus the diseases of "doctors" called for recourse to modern care structures and the diseases of "healers" called for recourse to traditional medicine. Thus the body of knowledge encompassing the disease at Widou is one of the reasons that has influenced care-seeking behavior. Each community (ethnic group, clan, village) has a body of knowledge about health that is constantly enriched or challenged by experience and will lead to behaviours that seek to optimize the survival and well-being of community members, according to its own rationalizations [17]. Similarly, each society has one or more nosologically and etiological systems that will enable it to generate therapeutic strategies according to the origin of the disease and the actors involved. Everywhere in West Africa, there is a dichotomy between "natural" diseases and diseases "caused" by witchcraft, geniuses, etc. In this study this dichotomy is found between the diseases called "Doctors" and those called "Healers". This classification of diseases into one or the other category varies according to societies and individual characteristics, such as education. It continues to guide therapeutic remedies. Diseases classified as natural or "Doctor" can be treated at the dispensary. In Widou they are diseases such as fever, they are treated at the post. But the diseases "caused", in Widou they are diseases of "Healers" are complicated according to traditional practitioners caused by mystical-religious entities and are above all magical treatments sometimes associated with medicinal plants. To classify the different models of explanation and treatment of the disease, Kleinman distinguishes three non-dissociated areas of therapeutic management: the popular, the traditional and the professional [18].

The forms of treatment are varied and depend on the nature of the disease, the therapist's specialty and the resources of the environment: the management of malaria can rely on incantations with magic words, amulets bearing magic inscriptions, massages, potions or enemas developed from the resources of the pharmacopoeia. The therapeutic secrets are always kept secret: insofar as it is commonly accepted that "each tree has its geniuses, its remedies and its spells", the precise knowledge of the virtues of the pharmacopoeia, its methods of harvest, preparation and administration remains secretly guarded [19]. There are different types of traditional medicines in Widou. These range from incantations, grey-grey to powders and plants. In Widou, plants and powders are the most used in traditional treatments these plants are used some with Koranic verses or with amulets such as cords. The nature of therapeutic practice also varies according to sociological factors: "the greater the political-religious power, and the more the activity of healer passes through speech (blessings) and transcendence (incantations); the weaker it is, on the contrary, and the more the action of healing relies on object (amulets) and substance" [20].

\subsection{The Quality of the Care-Patient Relationship, a Barrier to the Use of the Health Structure}

In Widou, one of the main problems relating to the accessibility of health services is the quality of the carepatient relationship. Indeed, it was recorded in Widou, that there were problems related to the behavior of providers 
considered unpleasant and responsible for poor quality reception. According to them; the unpleasant behaviors noted are out of step with the Fulani culture which advocates a certain affability. According to Formarier R (2007), we can understand and explain the gap that exists between the relational expectations of patients and families who have representations that are their own, stemming from their culture, their vision of health, their experience of illness and which make the care relationship a singular, unique relationship and the relational practices of caregivers who rely on collective representations, more or less pre-coded and which, in many cases, do not go beyond the interaction stage [21].

Generally speaking, the care-patient relationship influences the users' perception of the health structure. Conflicts with health personnel, had to be enumerated by some of the interviewees who claimed that these conflicting relationships were at the origin of the choice of other health structures as places of care for their children. These relations seem difficult in sub-Saharan African countries. A study concerning 5 sub-Saharan African countries highlights these difficult relations between carers and carers [22]. The patient's knowledge of the health workforce is often a determinant of how he or she is treated in the health care setting. Often, the patient is the victim of the anonymization of his condition [23]. Inadequate communication between patients and their caregivers causes problems in the use of health services [24]. In Africa, the "relational" dysfunctions in health services have unfortunately been attested by numerous observations. At the heart of the therapeutic act, some works stress that essential medical acts have not been performed as they should have been. Although known, at least theoretically, the prescribed standards and ideal behaviors are not implemented [25]. The relationship with the patient depends on many factors (personality of the caregiver and the patient, life course, pathology of the patient, context of care...) that each is unique [26]. Professional distance can be defined as "the moral and psychological limit to the expression of personal values within the framework of professional activity [27]. According to Prayez P (2009), there is a right distance, which he defines as "the capacity to be in contact with others « the difference of places"[28]. In addition to the stakes in relation to the carer and his emotional "self-protection", the search for the right professional distance also has an impact on the carer-caregiver relationship, and in particular on the establishment of a bond of trust. The more the caregiver and patient are in a healthy, fair relationship that respects their individualities and status, the better they will be able to build a relationship of trust. The familiarity developed can be at the origin of a transgression of professionalism necessary to the quality of care. In Widou, this familiarity between the carer and the looked after is partly at the origin of an underutilization of the health service by the populations.

Interviewees also reported problems with the availability of human resources. These were either physical availability or availability of the skills deemed necessary for health care.
Qualitative data analysis also identified other barriers to health service utilization. These are (i) drug discontinuation, especially for children's syrups, (ii) the high cost of health services for the purchase of medicines as a priority and (iii) geographical accessibility problems. Geographical accessibility problems were responsible for an increase in child health care costs through an increase in transport costs.

\section{Conclusion}

This study showed that disease representations play an important role in defining care-seeking behavior. Thus the 'singular representations' relating to the types of illness condition the type of recourse to care. The fact remains that there are characteristics specific to a type of remedy that may contribute to the underutilization of this type of remedy. In this specific case, it is the quality of the care-patient relationship that is judged to be of insufficient quality, which is a burden on the use of modern health services.

\section{Ethics}

The study protocol was submitted and validated by the ethics and health research committee of the Cheikh Anta Diop University of Dakar. The ethics committee validation number is $074 / 2015 / \mathrm{CER} / \mathrm{UCAD}$. The study team provided all necessary information related to the survey and confidentiality standards to respondents prior to conducting the survey. Collection agents informed potential participants that their decision to participate in the survey is entirely voluntary. The participant's oral or written consent was obtained prior to participating in the study. The participant had the flexibility to break off the interview at any time. In addition, for participants hampered by the use of an audio recorder, the device could be turned off and replaced by written notes.

A copy of the consent form was provided to each participant. The informed consent form had been verbally translated into the local language by the collection officer prior to the interview or discussion. The data collected was transcribed and kept confidential. The anonymity of the interviewees was respected during the transcript of the interviews

\section{Acknowledgements}

This work was cofunded by the Labex DRIIHM, French programme "Investissements d'Avenir" (ANR-11-LABX0010) which is managed by the ANR

\section{Authors Contributions}

Ndeye Mareme Sougou, Gilles Boetsch and Anta tal-Dia conceived and designed the study; Ndeye Mareme Sougou performed the field visits and data collection; Ndeye Mareme Sougou, Gilles Boetsch and Anta tal-Dia analyzed the data and wrote the paper. 


\section{Conflicts of Interest}

The authors declare no conflict of interest.

\section{References}

[1] Romero-Daza, N., (2002). 'Traditional Medicine in Africa. Annals of the American Academy of Political and Social Science, 583: 173-176.

[2] Janzen, J. (1995). La quête de la thérapie au bas-Zaïre. Paris: Karthala. 1995 - 287 pages - 1 Volumes.

[3] Saillant F. (1999), "Femmes, soins domestiques et espace thérapeutique". Revue Anthropologie et Sociétés, 23(2): 1539.

[4] Kleinman A. (1980), Patients and healers in the context of culture, Berkeley, University of California Press.

[5] Saillant F., "Femmes, soins domestiques et espace thérapeutique". Revue Anthropologie et Sociétés, 1999; 23(2): 15-39.

[6] Berche T. Anthropologie et santé publique en pays dogon par Thierry Berche. Préface de Jean-Pierre Olivier de Sardan, Paris, APAD-Karthala, 1998, 232 p, ISBN: 2-86537-814-4.

[7] Aubel J., Toure I., Diagne M. (2004) Senegalese grandmothers promote improved maternal and child nutrition practices: the guardians of traditions are not averse to change. Social Science \& Medicine.; 59: 945-959.

[8] Makinwa-Adebusoye, P., Sociocultural Factors Affecting Fertility in SubSaharan Africa. Population Division, Workshop on prospects for fertility decline in Hight fertility countries. 2001; New York.

[9] Petrie K J. and Weinman J. (1997), Perceptions of Health and Illness. Illness perceptions: a new paradigm for psychosomatics? Journal of Psychosomatic Research, Vol. 42, No. 2, pp. 113-116.

[10] Nkoma P P. Itinéraires thérapeutiques des malades au Cameroun: Les déterminants du recours à l'automédication. 7ème Conférence sur la Population Africaine: "Dividende Démographique en Afrique: Perspectives, Opportunités et Des”, Nov 2015, Johannesburg, Afrique du Sud. 2015.

[11] Weiss M. G., Sharma S. D. et al. (1986), Traditional concepts of mental disorder among indian psychiatric patients: Preliminary report of work in progress, Social Science and Medicine, 23, 379-86.

[12] Joly, P., Taïeb, O., Abbal, T., Baubet, T. \& Moro, M. (2005). Représentations culturelles, itinéraires thérapeutiques et santé mentale infantile en Guadeloupe. La psychiatrie de l'enfant, vol. 48, (2), 537-575. doi: 10. 3917/psye. 482. 0537.

[13] Fabrega H. (1977) Perceived illness and its treatment. A naturalistic study in social medicine. British Journal of Preventive Medicine; 31: 213-219.

[14] Massé R, Culture et Santé Publique. Les contributions de l'anthropologie à la prévention et à la promotion de la santé. Montréal-Paris: Gaëtan Morin, Éditeur, 1995, 499 pp.

[15] Fainzang S. (1986) L'intérieur des choses. Maladie, divination et reproduction sociale chez les Bisa du Burkina. L'Harmattan, pp. 205, 1986, Connaissance des hommes 29.

[16] Gruénais M E (1999), « Jaffré, Yannick \& Olivier de Sardan, Jean-Pierre, eds. - La construction sociale des maladies. Les entités nosologiques populaires en Afrique de l'Ouest. Paris, Presses universitaires de France, 374 p. (« Les champs de la santé »). », Cahiers d'études africaines [En ligne], $161 \mid 2001$, mis en ligne le 30 avril 2003, consulté le 24 décembre 2017.

[17] Cantrelle P, Locoh T. (1990) Facteurs culturels et sociaux de la sante en Afrique de l'ouest. LES Dossiers du CEPED N' 10 PARIS.

[18] Kleinman cite par Coppo P., Pisani L., Keita A. (1992) Perceived morbidity and health behaviour in a Dogon community. Social science and Medicine, 34 (11):1227-1235.

[19] Gessain M. (1979) Environnement africain: les usages des «guis» chez les Bassari. Cahiers d'étude du milieu et d'aménagement du territoire, Enda, 36-79.

[20] Fassin D. (1992) Pouvoir et maladie en Afrique. Anthropologie sociale dans la banlieue de Dakar. Paris: PUF, $359 \mathrm{p}$.

[21] Formarier, M. (2007). La relation de soin, concepts et finalités. Recherche en soins infirmiers, 89, (2), 33-42.

[22] Jaffré Y., Olivier de Sardan J.-P. (dir) -2001- Les dysfonctionnements des systèmes de soins. Enquête sur l'accès aux soins dans 5 capitales d'Afrique de l'Ouest. Projet « santé Urbaine »: Unicef, Coopération française, 290 p.

[23] Fainzang S. (2004) "Entre pratiques symboliques et recours thérapeutiques. Les problématiques d'un itinéraire de recherche". Anthropolitica, Revue d'anthropologie et de sciences politiques du Brésil, 15: 20-38.

[24] Altaras, R., Nuwa, A., Agaba, B., Streat, E., Tibenderana, J. K., Martin, S., \& Strachan, C. E. (2016). How Do Patients and Health Workers Interact around Malaria Rapid Diagnostic Testing, and How Are the Tests Experienced by Patients in Practice? A Qualitative Study in Western Uganda. PLoS ONE, 11(8):1-20. e0159525. doi:10.1371/journal.pone.015952

[25] Jaffré, Y. (2003). Le souci de l'autre: audit, éthique professionnelle et réflexivité des soignants en Guinée. Autrepart, 28, (4), 95-110.

[26] Barthel F. (2012). La distance professionnelle dans la relation soignant-soigné à domicile. Institut de Formation en Soins Infirmiers de Villeneuve-Saint-Georges Promotion.

[27] Martin M. (1997). La distance professionnelle, the international journal of applied philosophy, 11(2): 39-50.

[28] Prayez P., Sliwka C. (2009). Distance professionnelle et qualité du soin. France: Broché. Editions Lamarre. p. XVIII. p: 287. 